\title{
Numerical Computation of Unsteady Compressible Flow past a Plunging Aerofoil
}

\author{
K. S. Kumar ${ }^{\dagger}$ and S. V. Sajjan \\ Computational and Theoretical Fluid Dynamics Division, Council of Scientific and Industrial Research, National Aerospace \\ Laboratories, Bangalore - 560017, India \\ $\dagger$ Corresponding Author Email: sivakumar@nal.res.in
}

(Received June 7, 2010; accepted October 9, 2011)

\begin{abstract}
Unsteady Reynolds-averaged Navier-Stokes (RANS) computations are presented for subsonic and transonic flow past a plunging NACA 64A010 aerofoil. The Implicit RANS solver used for obtaining the time-accurate solution is based on finite volume nodal point spatial discretization scheme with dual time stepping. Results for the subsonic and transonic cases compare well with the experimental data, thus demonstrating the capability of the solver to provide useful unsteady pressure data for aeroelastic analysis.
\end{abstract}

Keywords: Viscous flow, RANS solver, Implicit method, Heaving aerofoil, Nodal point scheme.

\section{NOMENCLATURE}

\begin{tabular}{|c|c|}
\hline$A, B, R, S$ & Jacobian matrices \\
\hline$C_{d}$ & drag coefficient \\
\hline$C_{p}$ & surface pressure coefficient \\
\hline$C_{l}$ & lift coefficient \\
\hline$C_{m}$ & moment coefficient \\
\hline$F, G$ & inviscid flux vectors \\
\hline$M_{\infty}$ & free stream Mach number \\
\hline $\operatorname{Pr}$ & Prandtl number \\
\hline$P r_{t}$ & turbulent Prandtl number \\
\hline$R e_{\infty}$ & free stream Reynolds number \\
\hline$U$ & vector of conserved variables \\
\hline$U_{\infty}$ & free stream velocity \\
\hline$U^{n}$ & solution vector at time level $n$ \\
\hline$V, W$ & viscous flux vectors \\
\hline$c$ & aerofoil chord \\
\hline$e$ & energy \\
\hline $\begin{array}{c}\text { non- } \\
\text { plur }\end{array}$ & $\begin{array}{l}\text { nensional amplitude in } \\
\left(y_{0} / c\right)\end{array}$ \\
\hline$h_{i j}$ non-c & $\begin{array}{l}\text { area of quadrilateral } \\
\text { ensional reduced }\end{array}$ \\
\hline frequen & $\left(\omega c / 2 U_{\infty}\right)$ \\
\hline$n$ & time level \\
\hline$p$ & pressure \\
\hline$t$ & physical time \\
\hline
\end{tabular}

\section{INTRODUCTION}

The Navier-Stokes equations constitute the basic mathematical model for numerical simulation of compressible flows. They permit the analysis of complex flow phenomena such as shock/boundary layer interaction, flow separation, wake flow, hysteresis, periodic vortex shedding and so on. Since the

$\begin{array}{ll}t^{*} & \text { non-dimensional time }\left(U_{\infty} t / c\right) \\ u, v & \text { velocity components } \\ x, y & \text { Cartesian coordinates } \\ y_{0} & \text { amplitude of plunge or heave } \\ y(t) & \text { instant plunge distance of the } \\ & \text { aerofoil }\end{array}$

non-dimensional instant plunge

distance of the aerofoil

boundary curve

control volume surrounding the nodal point $(i, j)$ of

the curvilinear grid

$\begin{array}{ll}\Delta t & \text { real or physical time step } \\ \alpha_{o} & \begin{array}{l}\text { amplitude of pitching } \\ \text { oscillation }\end{array} \\ \alpha_{m} & \text { mean angle of attack } \\ \gamma & \text { ratio of specific heats } \\ \lambda, \mu & \text { viscosity coefficients } \\ \mu_{\infty} & \text { free stream viscosity coefficient } \\ \mu_{\mathrm{t}} & \text { turbulent viscosity coefficient } \\ \rho & \text { density } \\ \rho_{\infty} & \text { free stream density } \\ \omega & \text { non-dimensional angular frequency }\end{array}$

mathematical nature of the governing equations changes from elliptic in subsonic region to hyperbolic in supersonic region, compressible flow computations require sophisticated numerical techniques and enormous computational effort. Due to rapid advances achieved in numerical methods as well as computer technology, there has been considerable progress in the development of efficient Navier-Stokes solvers in the 
last two decades. Several finite difference and finite volume time stepping schemes have been applied successfully to a variety of two and three-dimensional fluid flow problems. Even though steady flow computations have attracted much attention, the focus has slowly shifted to unsteady flow computations in recent years [1].

Unsteady flows are encountered in many aerospace applications and prediction of unsteady air loads plays a vital role in aircraft and helicopter design (Mabey, 1999; McCroskey 1982; McCroskey, W. J. 1988). Since wind tunnel testing of unsteady flow situations is difficult and expensive, computational studies of wing stall and flutter, buffeting, gust response, dynamic stall, blade-vortex interaction of helicopter rotors etc. can provide important design data. Understanding these interesting unsteady flow phenomena through NavierStokes computations therefore presents a significant challenge for computational fluid dynamics.

The Implicit Reynolds-averaged Navier-Stokes Solver (IMPRANS) for simulating unsteady compressible flows over aerofoils and wings has been employed for solving the unsteady RANS equations on a moving mesh in an inertial frame of reference Vimala Dutta et al. (2003) The solver is based on an implicit finite volume nodal point scheme wherein a control volume is formed by joining the centroids of the neighbouring cells around a nodal point in the computational domain. The efficiency of the solver for making time-accurate computations is enhanced by implementing an implicit dual time stepping procedure.

In this approach, an equivalent pseudo steady state problem is solved at each real time step using local time stepping. The algebraic eddy viscosity model due to Baldwin and Lomax is used for turbulence closure Baldwin et al. (1978).

This solver has been extensively validated for computing unsteady flow past pitching aerofoils and wings Sharanappa et al. (2008) helicopter rotor blades Vimala Dutta et al. 2005; Sharanappa et al. 2006) and wind turbines Dutta et al. (2007). Plunging or heaving is a vertical displacement of the aerofoil perpendicular to the free stream direction. Most of the previous computational studies that have been published in literature are on plunging aerofoils for low speed flows, of interest to MAVs (Sarkar et al. 2006; Viieru et al. 2007; Andro et al. 2009; Ashraf et al. 2007). The present work is one of only a few to consider for unsteady flow past plunging aerofoil at subsonic and transonic Mach numbers.

\section{IMPRANS SOLVER}

The solver is based on an implicit finite volume nodal point spatial discretization scheme with dual time stepping. Inviscid flux vectors are calculated by using the flow variables at the six neighbouring points of hexahedral volume. Turbulence closure is achieved through the algebraic eddy viscosity model of Baldwin and Lomax.

\subsection{Governing Equations}

The two-dimensional Reynolds averaged Navier-Stokes equations for a moving domain can be written in nondimensional conservative form as

$\frac{\partial U}{\partial t}+\frac{\partial F}{\partial x}+\frac{\partial G}{\partial y}=\frac{\partial V}{\partial x}+\frac{\partial W}{\partial y}$

where

$U=\left[\begin{array}{l}\rho \\ \rho u \\ \rho v \\ e\end{array}\right], F=\left[\begin{array}{c}\rho\left(u-x_{t}\right) \\ \rho u\left(u-x_{t}\right)+p \\ \rho v\left(u-x_{t}\right) \\ e\left(u-x_{t}\right)+p u\end{array}\right], \quad G=\left[\begin{array}{c}\rho\left(v-y_{t}\right) \\ \rho u\left(v-y_{t}\right) \\ \rho v\left(v-y_{t}\right)+p \\ e\left(v-y_{t}\right)+p v\end{array}\right]$

$V=V_{1}\left(U, U_{x}\right)+V_{2}\left(U, U_{y}\right)$

$=\frac{1}{R e_{\infty}}\left[\begin{array}{c}0 \\ \lambda\left(u_{x}+v_{y}\right)+2 \mu u_{x} \\ \mu\left(v_{x}+u_{y}\right) \\ \mu v\left(u_{y}+v_{x}\right)+\lambda u\left(u_{x}+v_{y}\right)+2 \mu u u_{x}+\frac{\mu T_{x}}{(\gamma-1) M_{\infty}^{2} P r}\end{array}\right]$

$W=W_{1}\left(U, U_{x}\right)+W_{2}\left(U, U_{y}\right)$

$=\frac{1}{R e_{\infty}}\left[\begin{array}{c}0 \\ \mu\left(u_{y}+v_{x}\right) \\ \lambda\left(u_{x}+v_{y}\right)+2 \mu v \\ \mu u\left(u_{y}+v_{x}\right)+\lambda v\left(u_{x}+v_{y}\right)+2 \mu v v_{y}+\frac{\mu T_{y}}{(\gamma-1) M_{\infty}^{2} \operatorname{Pr}}\end{array}\right]$

Here $x$ and $y$ are the Cartesian coordinates and $t$ is the time variable; $x_{\mathrm{t}}$ and $y_{t}$ are the Cartesian velocity components of the moving domain. For a fixed domain, the grid speeds $x_{t}$ and $y_{t}$ are zero. $U$ is the vector of conserved variables; $F$ and $G$ are inviscid flux vectors and $V$ and $W$ are viscous flux vectors. The primitive variables are density $\rho$, velocity components $u, v$ in the $x$ and $y$ directions, pressure $p$, temperature $T$ and total energy $e$ per unit volume. The non-dimensional variables used in the above equations have been obtained by using the following free stream values as reference quantities: $\rho_{\infty}$ (density), $U_{\infty}$ (velocity), $\mu_{\infty}$ (viscosity), $\rho_{\infty} U^{2} \infty$ (pressure), $T_{\infty}$ (temperature), and so on. Some characteristic length such as chord $c$ of an aerofoil is chosen as the length scale.

$M_{\infty}$ and $R e_{\infty}$ are the free stream Mach number and Reynolds number respectively; $\gamma$ is the ratio of specific heats and $\mathrm{Pr}$ is the prandtl number. In addition, the viscosity coefficients $\lambda$ and $\mu$ are related by the Stokes relation

$3 \lambda+2 \mu=0$

and the Sutherland's law of viscosity is given by

$\mu=C_{1}\left[\frac{T^{3 / 2}}{T+C_{2}}\right]$

For turbulent flows, the laminar viscosity coefficient $\mu$ is replaced by, $\mu+\mu_{t}$ and $\mu / \operatorname{Pr}$ is replaced by $(\mu / P r)+$ $\left(\mu_{t} / P r_{t}\right)$; the turbulent viscosity coefficient $\mu_{t}$ and the turbulent Prandtl number $P r_{t}$ are provided by a 
turbulence model. Finally the system is closed using the perfect gas equation of state in non-dimensional form as

$$
P=\frac{\rho T}{\gamma M_{\infty}^{2}}
$$

The Euler equations for inviscid flow are obtained from the Navier-Stokes equations by setting

$$
1 / R e_{\infty}=0
$$

\subsection{Computational Method}

There are essentially two types of algorithms to solve unsteady compressible Reynolds-averaged NavierStokes equations, namely, explicit and implicit. In explicit schemes, the dependent variables at any new time-level are expressed in terms of variables at previous time steps and no matrix inversion is involved. Explicit determination of the dependent variables is not possible in implicit schemes and the difference equations at all grid points have to be solved simultaneously. In explicit methods, the time step imposed by numeric is much smaller than the time step that is required to resolve the physical unsteadiness of the flow problem. Even though explicit schemes are easy to implement, they need a very large number of time steps. Since convergence acceleration techniques like local time stepping used for steady flows destroy time accuracy and unsteady flow computations often need to be continued for a long time, the total CPU time requirement becomes very large. Implicit time discretization, on the other hand, allows much larger time steps and hence can be employed for time-accurate computations of flows of practical interest within reasonable CPU time. The computational work per time step to construct and solve the resulting matrix equations is, of course, larger than in an explicit scheme.

The use of integral forms of Euler/Navier-Stokes equations leads to the finite volume approach whereas the divergence form yields the finite difference approach. The main advantage of the finite volume method is the capability to handle arbitrary geometry through direct discretization in the physical space. In cell-centered finite volume schemes, the dependent variables are associated with the centre of the cell in the computational mesh. In cell-vertex or nodal point schemes, the flow quantities are specified at cell vertices rather than at cell centre's. With a view to implement the cell-vertex finite volume approach to implicit schemes, an implicit finite volume nodal point schemes has been developed at National Aerospace Laboratories (NAL). The numerical scheme for solving the two-dimensional Navier-Stokes equations governing the viscous compressible flow over aerofoils has been derived by using Euler's time differencing formula with nodal point discretization. Certain basic ideas from implicit finite difference scheme of Beam et al. (1978) and Steger (1978), the nodal point schemes of $\mathrm{Ni}$ (1982) and Hall (1985), the Runge kutta timestepping scheme of Jameson et al. (1981) and cellcentered schemes due to Hollanders et al. (1980) Hollanders et al. (1985) have been combined efficiently in the present method.

Applying Euler's implicit time differencing formula
$U^{n}=U^{n+1}-\left(\frac{\partial U}{\partial t}\right)^{n+1} \Delta t+O\left(\Delta t^{2}\right)$

to the governing Eq. (1), we obtain

$\Delta U^{n}+\Delta t\left[\frac{\partial}{\partial x}(F-V)+\frac{\partial}{\partial y}(G-W)\right]^{n+1}=0$

Here $U^{n}=U(t)=U(n \Delta t)$ is the solution vector at time level $n$ and $\Delta U^{n}=\left(U^{n+1}-U^{n}\right)$ is the change in $U^{n}$ over time step $\Delta t$. In order to facilitate the finite volume formulation, the above equations are written in the integral form as

$\iint_{\Omega} \Delta U^{n} d x d y+\Delta t \int_{\Gamma}\left[(F-V)^{n+1} d y-(G-W)^{n+1} d x\right]=0$

where $\Omega$ is any two-dimensional flow domain and $\Gamma$ is the boundary curve.

In the nodal point finite volume approach (Ni 1982 and Hall 1985), the flow variables are associated with each mesh point of the grid and the integral conservative equations are applied to each control volume obtained by joining the centroids of the four neighbouring cells of a nodal point. Fig. 1(a) shows a typical control volume $\Omega_{i j}$ formed by joining the centres $a, b, c, d$ of the four cells surrounding a nodal point $(i, j)$ of a body fitted curvilinear grid, $i, j$ being the spatial indices along curvilinear coordinate directions $\xi$, $\eta$. Application of nodal point spatial discretization to Eq. (10) leads to the following equations for the computational cell $\Omega_{i j}$

$\Delta U_{i j}^{n} h_{i j}+\Delta t \int_{\Gamma_{i j}}\left[(F-V)^{n+1} d y-(G-W)^{n+1} d x\right]=0$

where $h_{i j}$ is the area of quadrilateral abcd and the integral refers to a contour integration around the boundary $\Gamma_{i j}$ of the cell $\Omega_{i j}$ in anticlockwise direction. The fluxes are calculated across the four sides of the control volume $a b c d$.

Linearising the changes in flux vectors using Taylor's series expansions in time and assuming locally constant transport properties, Eq. (11), can be simplified to

$$
\begin{aligned}
& \Delta U_{i j}^{n}+\frac{\Delta t}{h_{i j}}\left[\int_{\Gamma_{i j}}\left\{A^{n}-\frac{\partial}{\partial x} R^{n}\right\} \Delta U^{n} d y-\int_{\Gamma_{i j}}\left\{B^{n}-\frac{\partial}{\partial y} S^{n}\right\} \Delta U^{n} d x\right] \\
& =-\frac{\Delta t}{h_{i j}}\left[\int_{\Gamma_{i j}}(F-V)^{n} d y+\int_{\Gamma_{i j}}(G-W)^{n} d x\right]
\end{aligned}
$$

Here $A, B, R$ and $S$ are the Jacobian matrices given by

$A=\frac{\partial F}{\partial U}, B=\frac{\partial G}{\partial U}, R=\frac{\partial V_{1}}{\partial U_{x}}$ and $S=\frac{\partial W_{2}}{\partial U_{y}}$

\subsection{Moving Aerofoil's}

For simulating unsteady flow over moving aerofoils, the governing equations are solved in an inertial frame of reference. The flow field is discretized by employing a $\mathrm{C}$-grid which is fixed to the aerofoil so that the computational grid translates and rotates rigidly with the aerofoil. For any grid point $\mathrm{z}=x+i y$ in the moving domain fixed to the aerofoil which is shown in Fig. 1(b).

$$
\begin{aligned}
& \left(z-z_{c}\right) e^{i \alpha}=\text { Constant; } \\
& \dot{z}=\dot{z}_{c}-i \dot{\alpha}\left(z-z_{c}\right) \\
& \ddot{z}=\ddot{z} c-(\dot{\alpha}+i \ddot{\alpha})\left(z-z_{c}\right)
\end{aligned}
$$


The nodal points $(x, y)$ and the grid speeds $\left(x_{t}, y_{t}\right)$ at any particular instant are computed from the prescribed aerofoil motion, thus eliminating the need for multiple grid generation. Along the moving body surface, conditions for zero relative velocity and adiabatic wall are imposed. The steady solution at initial incidence is used as the starting solution.

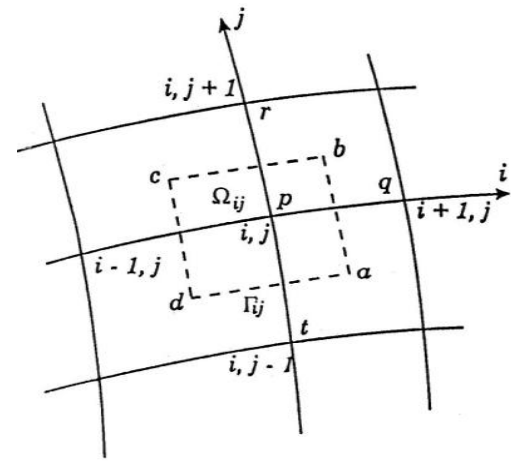

Fig. 1(a). A typical control volume

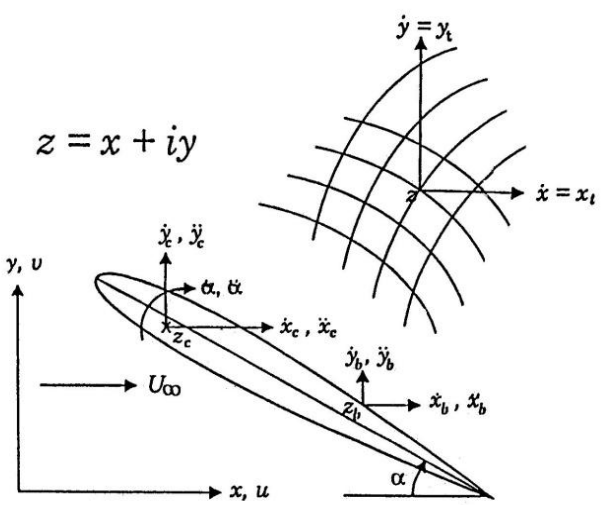

Fig. 1(b). Moving aerofoil configuration

\section{GRID GENERATION}

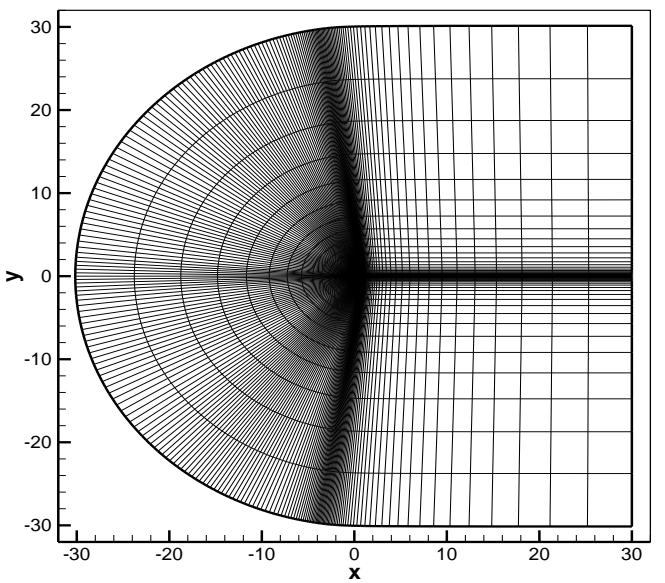

Fig. 2(a).C- Grid around the aerofoil

The structured C-grid with a grid size of 247 x 65 (streamwise $\mathrm{x}$ normal) is generated around the NACA 64A010 aerofoil, as shown in Fig. 2(a). Here, 167 points are distributed on the aerofoil surface and 41 points in the wake region. The outer boundary is located 30 chords away from the aerofoil surface. The first grid spacing on the aerofoil surface is $0.00001 \mathrm{c}$ in the direction normal to the aerofoil surface. The grid points are properly clustered near the leading and trailing edges and the wall. The close-up view of the grid is shown in Fig. 2(b).

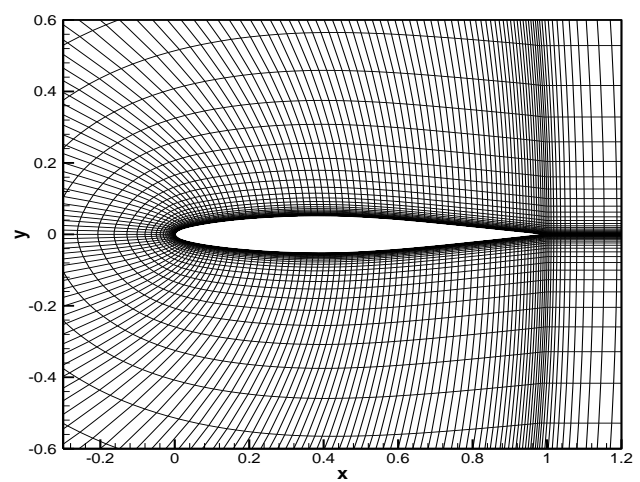

Fig. 2(b). Close-up view of the grid

\section{Results}

The computation is carried out for two-dimensional unsteady compressible viscous flow over a plunging aerofoil at subsonic and transonic Mach numbers, where sinusoidal plunging or heaving motion of the aerofoil is given by

$y(t)=y 0 \cos (\omega t)$.

Here $y 0$ is the amplitude in plunge, $\omega$ is the frequency of oscillation, c is the aerofoil chord length and $U_{\infty}$ is the free stream velocity. When all the parameters are non-dimensionalized with respect to c and $U \infty$, the time, amplitude in plunge and reduced frequency can be defined as $t^{*}=U \infty t / c, h a=y 0 / c$ and $k=\omega c / 2 U \infty$. The non-dimensional displacement of the input motion is given by the expression,

$y\left(t^{*}\right)=h a \cos \left(2 k t^{*}\right)$

The time-accurate results are presented for flow around the aerofoil undergoing small amplitude heave motion corresponding to the experiments of Davis et al. (1980) The input parameters used for the present computations are listed in Table 1 for the NACA 64A010 aerofoil. The unsteady variation of non-dimensional plunge displacement of the aerofoil, with non-dimensional time $\left(t^{*}\right)$ for five cycles of heaving oscillation is shown in Fig. 3.

The first harmonic components of unsteady surface pressure data have been evaluated through a Fourierseries representation of the surface pressure coefficient $C_{p}$. The mean values and the Fourier coefficients corresponding to real and imaginary parts of the first harmonics are plotted in Fig. 4. The mean and fundamental frequency pressure data show good agreement between computations and the experiments of Davis and Malcolm Davis et al. (1980). The presence of shocks on both the surfaces of the aerofoil and their strength and location are well predicted in the transonic flow regime. 

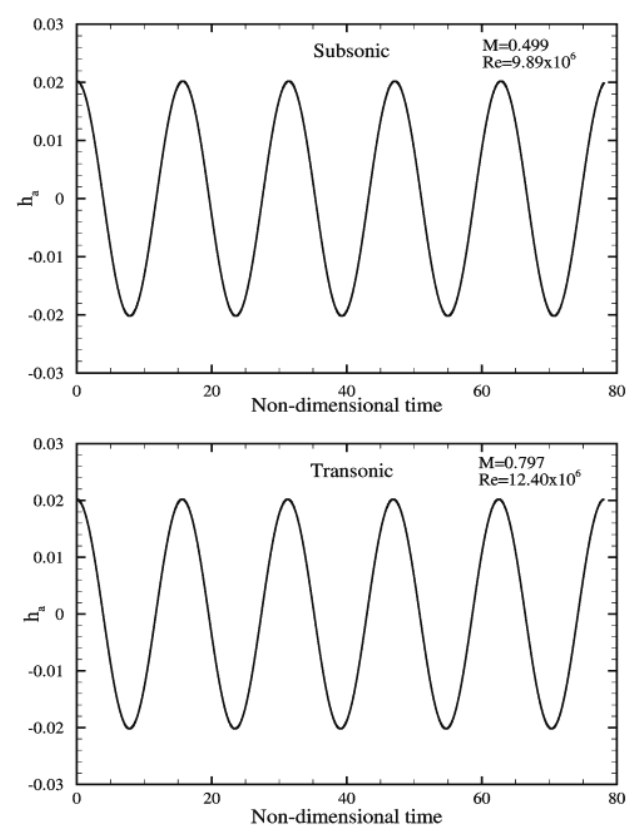

Fig. 3. Unsteady variation of heave amplitude with time on plunging NACA 64A010 aerofoil for subsonic and transonic cases.
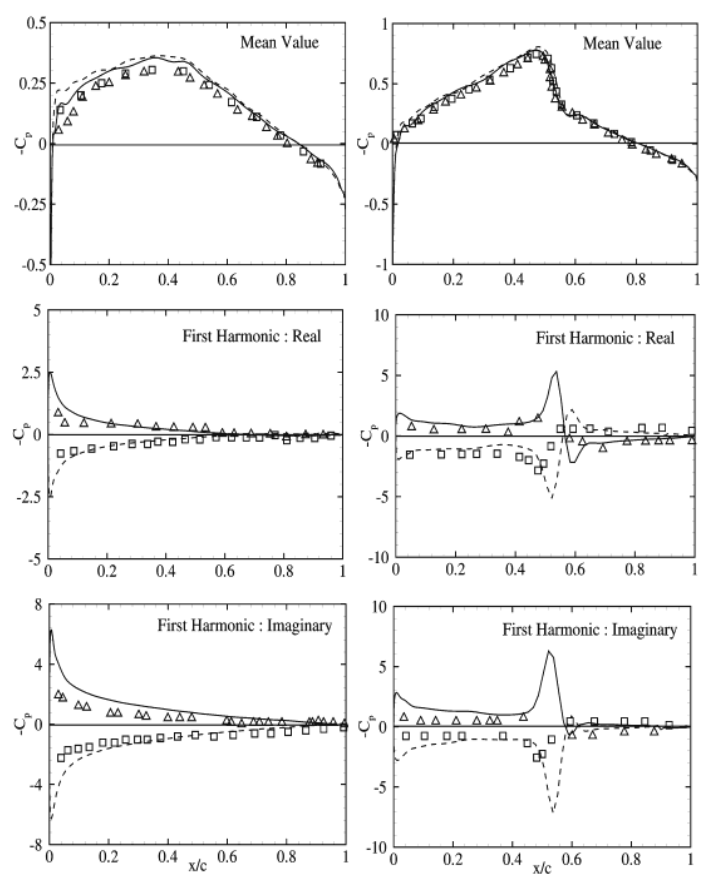

Fig. 4. Unsteady surface pressure distribution for subsonic (left) and transonic (right) cases. (Experiment

\section{Davis et al.(1980): $\rightarrow$ Upper; $\Delta \rightarrow$ Lower}

Computation: $\rightarrow$ Upper; $-\rightarrow$ Lower)

The time histories of $C_{l}, C_{d}$ and $C_{m}$ for five cycles of heaving motion are plotted in Fig. 5 , for both the cases. The aerodynamic loads attain a periodic behavior after four cycles of oscillations. The unsteady variation of lift and moment coefficients with heave distance is shown in Fig. 6, for both subsonic and transonic cases. The computed loops of the aerodynamic coefficients clearly demonstrate the hysteretic property existing between the up-stroke and down-stroke motion of the aerofoil. The unsteady surface pressure coefficient distribution on plunging aerofoil for subsonic and transonic cases for one complete cycle at approximately equal time intervals are shown in Fig. 7 and Fig. 8 respectively. The Mach number contours are plotted in Fig. 9 and Fig.10 for both the cases respectively. The Mach number contour plots and the surface pressure distribution plots show that in transonic case, there is a strong shock on the upper surface that weakens when it reaches the upstream position during upward stroke of the aerofoil similarly there is a strong shock on the lower surface that becomes weak when it reaches upstream position during downward stroke of the aerofoil. In both cases, plunging motion of the aerofoil creates asymmetry in the flow field even for symmetric aerofoils. This leads to different pressure fields on the upper and lower surfaces and increases the lift compared to the static aerofoils.
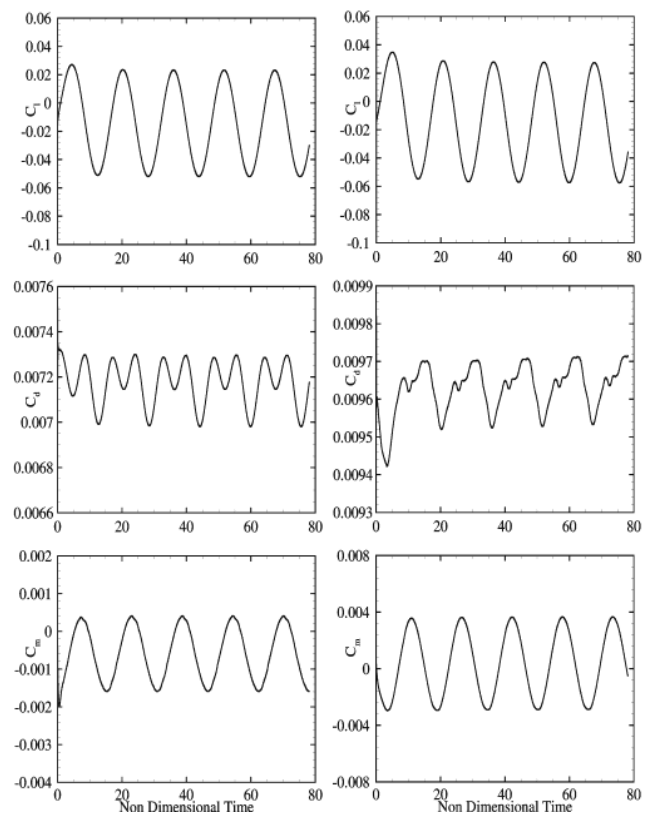

Fig. 5. Time histories of $C_{l}, C_{d}$ and $C_{m}$ for subsonic (left) and transonic (right) cases.
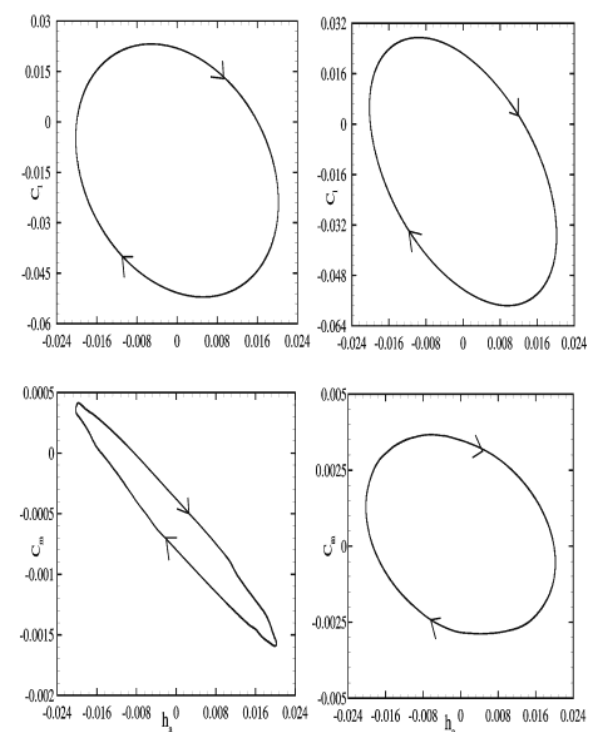

Fig. 6.Unsteady variations of lift and moment coefficients with heave distance for subsonic (left) and transonic (right) cases 

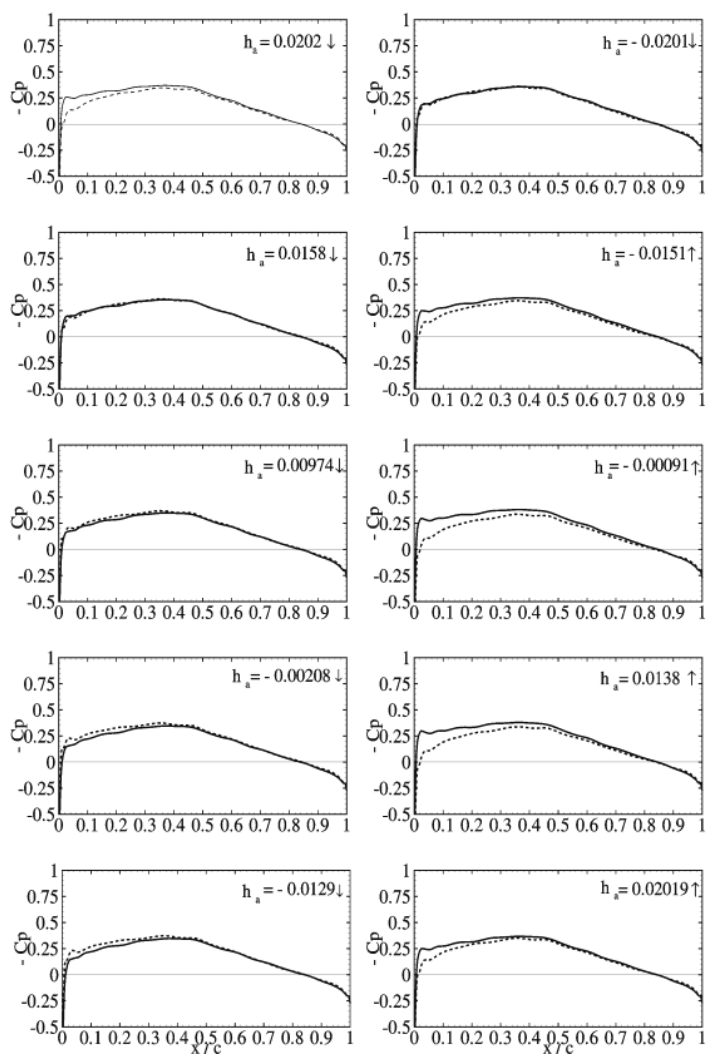

Fig. 7. Unsteady surface pressure distribution during one complete cycle for subsonic case.

$(---\rightarrow$ Upper surface, $-\rightarrow$ Lower surface)
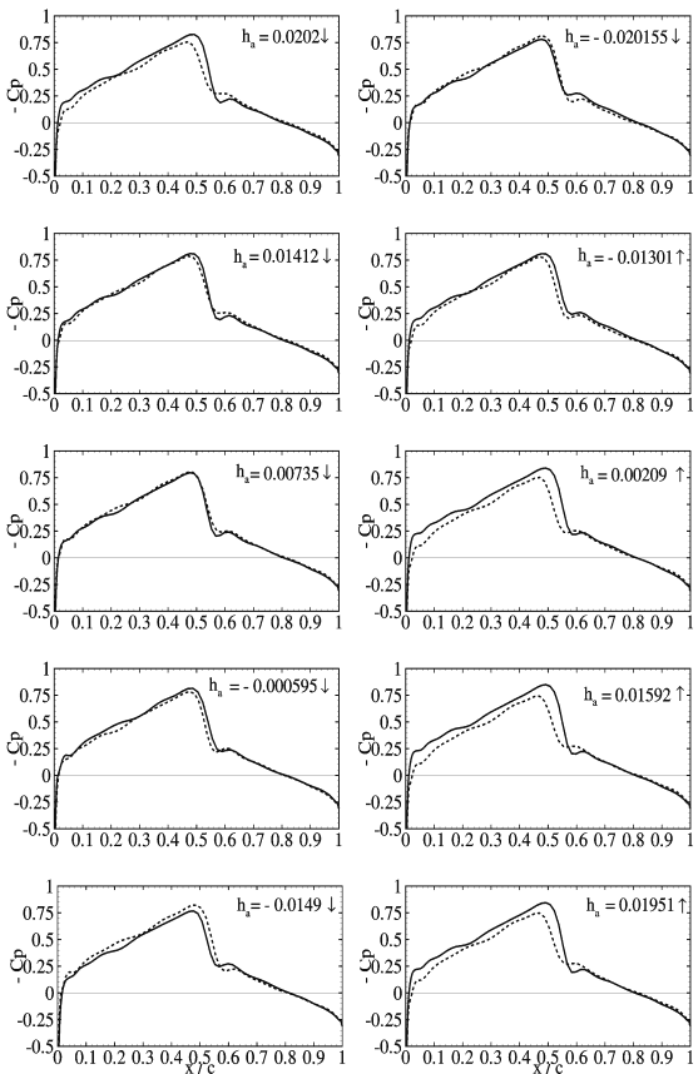

Fig. 8. Unsteady surface pressure distribution during one complete cycle for transonic case. $(---\rightarrow$ Upper surface, $-\rightarrow$ Lower surface)

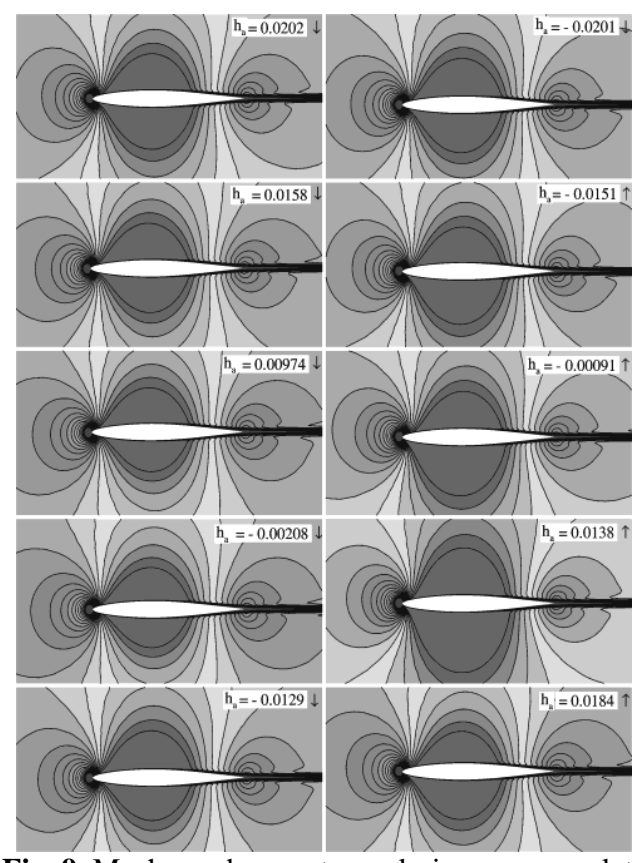

Fig. 9. Mach number contours during one complete cycle for subsonic case.

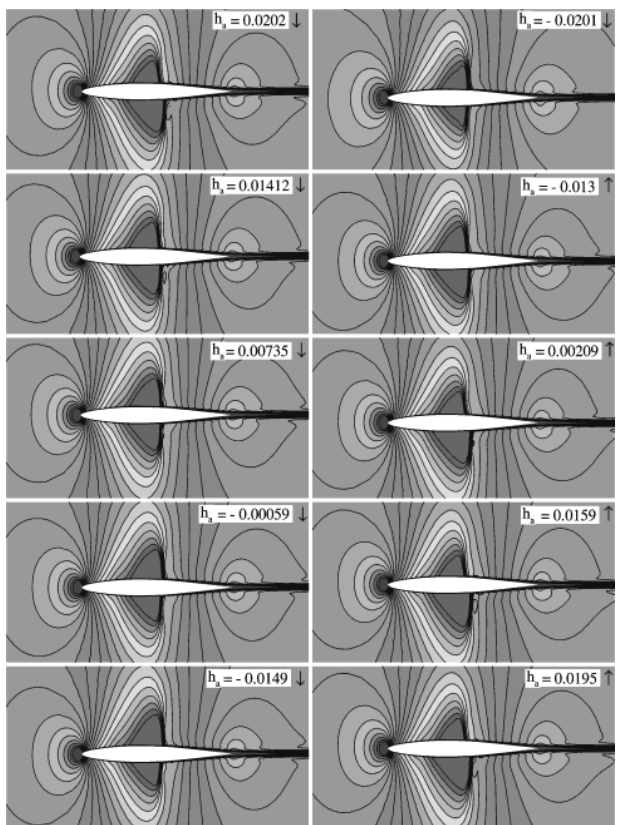

Fig. 10. Mach number contours during one complete cycle for transonic case.

Table 1 Unsteady aerodynamic input flow conditions used for plunging NACA 64A010 aerofoil

\begin{tabular}{|c|c|c|}
\hline $\begin{array}{c}\text { Input } \\
\text { parameter }\end{array}$ & Subsonic case & Transonic case \\
\hline$M_{\infty}$ & 0.499 & 0.797 \\
\hline$R e_{\infty}$ & $9.89 \times 10^{6}$ & $12.40 \times 10^{6}$ \\
\hline$\alpha_{m}$ & $-0.13^{0}$ & $-0.08^{0}$ \\
\hline$k=\omega c / 2 U_{\infty}$ & 0.20 & 0.201 \\
\hline$h_{a}$ & 0.0202 & 0.0202 \\
\hline
\end{tabular}




\section{CONCLUSION}

The applicability of the Implicit Reynolds-averaged Navier-Stokes Solver (IMPRANS) for aeroelastic studies is illustrated through time accurate computations for two-dimensional subsonic and transonic flow past a plunging aerofoil. The unsteady aerodynamic data obtained for NACA 64A010 aerofoil is validated with the available experimental results. The plunging motion of the aerofoil creates asymmetry in the flow field even for symmetric aerofoil's.

This leads to different pressure fields on the upper and lower surfaces and increases the lift compared to the static aerofoils. In transonic case, there is a strong shock on the upper surface that weakens when it reaches the upstream position during upward stroke of the aerofoil similarly there is a strong shock on the lower surface that becomes weak when it reaches upstream position during downward stroke of the aerofoil.

\section{ACKNOWLEDGEMENTS}

The authors gratefully acknowledge Dr. P. K. Dutta and Dr. Vimala Dutta for their constant encouragement and support during the work.

\section{REFERENCES}

Unsteady aerodynamics (1996, July). Procee-dings Two day Conference Royal Aero-nautical Society, London.

Mabey, D. G. (1999, January). Unsteady Aerodynamics: Restrospect and prospect. Aeronautical Journal, 103(1019), Review Paper No. 003, $1-18$.

McCroskey, W. J. (1982). Unsteady airfoils. Ann. Rev. Fluid Mech., Vol. 14, 285 - 311

McCroskey, W. J. (1988, March). Some Rotorcraft Applications of Computational Fluid Dynamics. NASA TM 100066.

Vimala Dutta, P. K., Dutta and Sharanappa (2003, March). An Implicit RANS Solver for Unsteady Compressible Flow Compu-tations. In Proceedings of the Seminar on 'State of the Art and Future Trends of CFD at NAL', NAL SP 0301 .

Baldwin, B. S. and H. Lomax, (1978). Thin Layer approximation and algebraic model for separate turbulent flows, AIAA Paper No. 78 - 257.

Sharanappa V., Sajjan, Vimala Dutta and P. K. Dutta (2008, August). Numerical Simu-lation of flow over pitching bodies using an implicit Reynoldsaveraged Navier-Stokes solver. In Procedings of $12^{\text {th }}$ Asian congress of Fluid Mechanics, Daejeon, Korea.
Vimala Dutta, Sharanappa and P. K. Dutta (2005, August). Navier-Stokes Compu-tations for a Helicopter Rotor Blade in Hover. In Proceedings Eighth Annual CFD Symposium, CFD Division of Aeronautical Society of India, Bangalore, CP 18.

Sharanappa V., Sajjan, Vimala Dutta and P. K. Dutta (2006 August). Viscous Unsteady Flow Around a Helicopter Rotor Blade in Forward Flight. In Proceedings $9^{\text {th }}$ Annual CFD symposium, CFD Division of Aero-nautical Society of India, Bangalore.

Dutta, P. K., Vimala Dutta and Sharanappa V., Sajjan (2007, November). RANS Compu-tation of Flow Past Wind Turbine Blades. In Proceedings of $7^{\text {th }}$ Asian Computational Fluid Dynamics Conference, Bangalore, $26^{\text {th }}-30^{\text {th }}$, (Invited Paper).

Sunetra Sarkar and Karthik Venkatraman (2006). Numerical Simulation of in-compressible viscous flow past heaving airfoil. International Journal for Numerical Methods in fluids, Vol. 51, 1 - 29.

Jian Tang Dragos Viieru and Wei Shyy (2007, January). Effects of Reynolds Number and Flapping Kinematics on Hovering Aero-dynamics. 45th AIAA Aerospace Sciences Meeting and Exhibit, Reno, Nevada.

Jean-Yves Andro and Laurent Jacquin (2009). Frequency effects on the aerodynamic mechanisms of a heaving airfoil in a forward flight configuration. Aerospace Science and Technology $13,71-80$.

Ashraf, M. A., J. C. S Lai and J. Young, (2007, December). Numerical Analysis of Flapping Wing Aerodynamics. $16^{\text {th }}$ Australasian Fluid Mechanics Conference, Crown Plaza, Gold Coast, Australia.

Beam, R. M. and R. F. Warming, (1978, April). An Implicit Factored Scheme for the Comp-ressible Navier-Stokes Equations. AIAAJ, 16( 4), 393 402.

Steger, J. L. (1978). Implicit Finite Difference Simulation of flow about Arbitrary Geo-metries with application to airfoils. AIAAJ, Vol. 16, pp. $679-686$

Ni, R. H. (1982). A Multiple Grid Scheme for Solving the Euler Equations. AIAAJ, Vol. 20, 1565 - 1571.

Hall, M. G. (1985, April). Cell Vertex Multigrid Scheme for Solution of the Euler Equations. RAETM-Aero 2029, In Proceedings Conf. on Numerical methods for fluid dynamics, 303 - 345.

Jameson, A. W., Schmidt and E. Turkel (1981). Numerical Solution of Euler Equations by Finite Volume Methods Using Runge Kutta Time Stepping Schemes. AIAA Paper 81 - 1259. 
K. Siva Kumar et al. / JAFM, Vol. 5, No. 4, pp. 131-138, 2012.

Hollanders, H. and H. Viviand, (1980). The Numerical Treatment of Compressible High Reynolds Number Flows. Computational Fluid Dynamics, Vol. 2, Kollmann, W.,(ed), Hemisphere Publishing Corporation, 1 - 65.

Hollanders, H., A. Lerat and R. Peryret, (1985, November). Three-Dimensional Calculation of Transonic Viscous Flows by an Implicit method. AIAAJ, 23(11) 1670 - 1678.

Davis, S. S. and G. Malcolm (1980, August). Experimental Unsteady Aerodynamics of Conventional and Supercritical Aerofoils. NASA $\mathrm{TM}-81221$. 